\title{
Adverse Reactions and Resistance of Anticancer Drug Cisplatin
}

\author{
Zhonglie Yang, Li Lin, Xiaoyan Peng, Xinyi Yu, Xiaobin Zhang, Hezhong Jiang, and Jiahong Li* \\ School of Life Science and Engineering, Southwest Jiaotong University, Chengdu, Sichuan 614202, China \\ Email: jiahongljh@163.com (J. L.)
}

\begin{abstract}
Since 1978, cisplatin has become a leading anticancer drug for its high efficacy and unique mechanism of action. As a representative of platinum anticancer drugs, cisplatin has an excellent curative effect and broad application range of cancer. However, its application is limited by high toxicity and drug resistance. This review mainly focuses on adverse reactions and drug resistance of cisplatin.
\end{abstract}

Keywords cisplatin, anticancer, adverse reaction, resistance

\section{Introduction}

In 1844, Italian chemist Michele Peyrone has firstly prepared cisplatin, once known as the Peyrone's salt, but it did not attract the attention of scientists. ${ }^{[1]}$ In 1891 , Werner, the founder of modern coordination chemistry, established the coordination theory based on the study of this compound, and clarified the cis structure of Peyrone's salt. ${ }^{[2]}$ It was not until 1960 s that Rosenberg discovered that platinum complexes could inhibit cell division. ${ }^{[3]}$ Subsequent experiments proved that cisplatin played an essential role in the process, arousing him to make a bold guess: cisplatin had anticancer activity. It is proved that cisplatin possessed a good anticancer effect through anticancer test, arousing people's interest in the pharmacology of platinum complexes. In 1971, cisplatin was first employed in the treatment of cancer patients. ${ }^{[4]}$ In December 1978, the U.S. Food and Drug Administration (FDA) approved cisplatin for clinical use and as a commodity supply market.

cis-Diamminedichloroplatinum (II) (cis-[ $\left.\left.\mathrm{PtCl}_{2}\left(\mathrm{NH}_{3}\right)_{2}\right], 1\right)$, so called cisplatin, is centered on platinum atom, which forms a planar quadrilateral compound with two chlorine atoms on the same side and two ammonia atoms (Figure 1). The main pharmacological action of cisplatin is platinum atom, and only cis has anticancer pharmacological activity.<smiles>N[PH](N)(Cl)Cl</smiles>

Figure 1 The structure of cis-diamminedichloroplatinum(II) (1).

As early as 1973, Cleare and Hoeschele have clarified the structure-activity relationship of cisplatin, which is of great significance to explore the mechanism of action and drug resistance of cisplatin. ${ }^{[5,6]}$ Later, according to the structureactivity relationship of cisplatin, a large number of platinum anticancer drugs with low toxicity and high efficiency in the treatment of various cancers were discovered correspondingly. For instance, anti-tumor mechanism of cisplatin is based on the fact that nucleophilic amino groups formed by its hydrolysis can provide cisplatin-DNA complex with the DNA of tumor cells, thus preventing tumor cells from replicating and playing an anti-tumor role. Among them, a large amount of oxygen free radicals are provided in the hydration process of cisplatin, which is one of the important reasons for nephrotoxicity. According to the chemical structure of cisplatin, leaving group chlorine plays an important role in the hydration rate of cisplatin, which means that chlorine has an important influence on the final oxygen free radicals. Given the leaving group difference between cisplatin and carboplatin, carboplatin has no obvious nephrotoxicity. It is speculated that the leaving group chlorine may be one of the causes of cisplatin-induced nephrotoxicity.

As a non-specific drug of cell cycle, cisplatin has distinct cytotoxicity. Because cancer cells proliferate and synthesize more rapidly than normal cells, cancer cells are more sensitive to the cytotoxicity of cisplatin. Once cisplatin enters the cells, it first hydrates with chloride ions, and the attack of hydrated cisplatin on the intracellular target is significantly enhanced. Although cisplatin can interact with many cell components, DNA is the primary target of cisplatin. ${ }^{[7]}$ The anticancer effect of cisplatin is mainly due to the combination of $\mathrm{Pt}$ (II) in cisplatin with DNA bases in cancer cell nucleus along with allosteric DNA forming several adducts. Then DNA cannot be employed as a normal replication template for replication, giving rise to DNA replication disorder and inhibiting the division and proliferation of tumor cells. ${ }^{[8]}$ Briefly speaking, cisplatin can inhibit the DNA replication process of cancer cells and damage the structure of cell membrane, which has a strong broad-spectrum anti-cancer effect. The efficacy of cisplatin has undergone more than 40 years of clinical verification, till today, it is still widely used in the treatment of cancer, such as cervical cancer, ovarian cancer, bladder cancer, testicular cancer, head and neck cancer, non-small-cell lung cancer (NSCLC) and mesothelioma. ${ }^{\left[{ }^{9]}\right.}$

Cisplatin's high efficiency is accompanied by high toxicity, which is manifested in reproductive toxicity, genotoxicity and carcinogenicity. In view of this, carboplatin (2), the secondgeneration platinum anticancer drug, has been developed. Compared with the first generation of cisplatin, carboplatin has lower side effects and longer lasting action. ${ }^{[10]}$ However, carboplatin and cisplatin have cross resistance. Subsequently, oxaliplatin (3), the third-generation platinum anticancer drug, was approved by FDA. Oxaliplatin maintains the cis-structure of cisplatin, with platinum as the center, diaminocyclohexane with large steric hindrance as the retention ligand, and oxalate group as the leaving group. The introduction of diaminocyclohexane ligands overcomes the drug resistance of tumor cells owing 

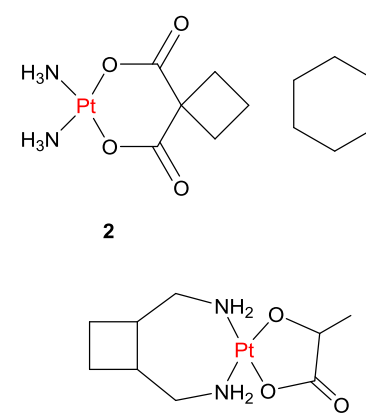<smiles>O=C1O[PH]2(N[C@H]3CCCC[C@H]3N2)OC1=O</smiles>

3

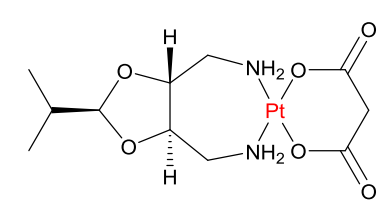

6

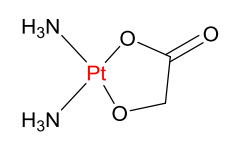

Figure 2 The structures of carboplatin (2), oxaliplatin (3), nedaplatin (4), lobaplatin (5), and heptaplatin (6).

to the fact that diaminocyclohexane with a large steric hindrance can prevent repair proteins from binding to DNA, ${ }^{[1]}$ thus promoting the death of cisplatin-resistant tumor cells. Therefore, oxaliplatin became the first platinum drug to resist drug resistance of tumor cells. Nedaplatin (4), first approved to be marketed in Japan in 1995, is a second-generation platinum antineoplastic drug developed by Shionogi \& Co., Ltd. for the treatment of head and neck tumors, small cell and non-small cell lung cancer, esophageal cancer, bladder cancer, testicular cancer, cervical cancer, and so on. In addition, lobaplatin (5) for cancer treatment in China and heptaplatin (6) for cancer treatment in the Republic of Korea ${ }^{[12]}$ have been approved, too.

\section{Adverse Reactions of Cisplatin}

Although cisplatin is effective against a variety of cancers as an anticancer drug, it also has obvious side effects owing to the fact that cisplatin's action is nonspecific, and targets random DNA regions. One of the limitations of cisplatin chemotherapy is the development of dose-limiting toxicity, preventing the continuation of treatment. ${ }^{[12]}$ Nephrotoxicity, ototoxicity, neurotoxicity and gastrointestinal reactions are considered as the most common clinical manifestations of adverse reactions.

\section{Nephrotoxicity}

There are two pathological mechanisms of cisplatin nephrotoxicity: one is that cisplatin can cause vasoconstriction along with the decrease of renal blood flow and glomerular filtration rate; the other is that cisplatin can cause ischemia, hypoxia and even necrosis of proximal tubular epithelial cells. Cisplatin nephrotoxicity can be caused by platinum accumulation, cytotoxicity and oxidative damage in kidney. Cisplatin is mainly administered intravenously by artery or radiation. After intravenous administration, cisplatin binds to plasma protein and distributes to all tissues and organs with blood circulation. About five hours after administration, half of the patients had a rise in drug blood concentration, which was related to the tissue reabsorption and release of cisplatin as well as hepatointestinal circulation. Furthermore, due to the release and binding of cisplatin to tissue and plasma proteins, the elimination of cisplatin in vivo is very slow, subsequently, giving rise to graver organ damage. The concentration of cisplatin in kidney is the highest, and the concentration of renal tubule is the highest in kidney. There is no biotransformation of cisplatin in vivo, and most of cisplatin is excreted through the kidney in the form of original form or binding to DNA.

Approximately one-third of patients reported nephrotoxicity during treatment with cisplatin. ${ }^{[13]}$ Compared with young people, elderly people are more likely to undergo nephrotoxicity. Owing to the fact that the incidence rate of cancer will increase up to $67 \%$ in the elderly from 2010 to 2030 , we should consider more about the kidney damage caused by cisplatin in the elderly. ${ }^{[14]}$ With the increase of single dose and treatment course of cisplatin, aging may cut down cisplatin clearance rate and ascend the concentration of serum ultrafilterable cisplatin, which could aggravate tubulointerstitial injury based on age. ${ }^{[15]}$ Dose plays an important role in nephrotoxicity, moreover, it was found that a dose of $40 \mathrm{mg} / \mathrm{m}^{2}$ caused severe renal damage. ${ }^{[15]}$ But recent studies showed that the variation of doses that could cause nephrotoxicity in risk groups is large, from 26.58 to $120.61 \mathrm{mg} / \mathrm{m}^{2}$, with a median of $71.74 \mathrm{mg} / \mathrm{m}^{2}$. ${ }^{[16]}$ After a single large dose of cisplatin, slight and reversible renal dysfunction may occur along with microalbuminuria. Repeated high-dose and short-term repeated use of drugs could bring about irreversible renal dysfunction, severe renal tubular necrosis, resulting in anuria and uremia. To avoid this from happening, hydration and diuresis must be carried out at the same time when a large dose is applied. Hydration therapy is a treatment method of hydration, increasing the amount of chlorine in urine to moderate renal toxicity. ${ }^{[17]}$

Cisplatin combined with other drugs has been employed in many treatment cases to alleviate nephrotoxicity, such as OCT2 inhibitor cimetidine, antioxidant carvedilol and cytoprotectant amifostine. However, recent in vitro data showed that ondansetron had a more potent inhibition toward MATEs than OCT2, suggesting that ondansetron may cause more serious nephrotoxicity. ${ }^{[18]}$ The latest research showed that Aprepitant, ${ }^{[19]}$ Citrus limoniaoil, ${ }^{[20]}$ L-tetrahydropalmatine, ${ }^{[21]}$ alpha-lipoic acid (ALA) and coenzyme Q10 (CoQ10) ${ }^{[22]}$ could attenuate or treat cisplatin-induced nephrotoxicity. However, most of drugs used for protective measures are limited to in vitro cells or animal experimental models.

\section{Ototoxicity}

The incidence of hearing loss caused by cisplatin is high, about $11 \%-97 \%$ in adults and more than $60 \%$ in average..$^{[23]}$ Inflammation is the first event triggered by exposure to cisplatin, followed by a series of reactions leading to oxidative stress and reactive oxygen species (ROS), which eventually brings about hearing loss. ${ }^{[2]}$ By comparison, the risk of hearing loss in children is significantly increased. ${ }^{[25]}$ The incidence of ototoxicity is closely related to high frequency and high dose of cisplatin. It is worth noting that hearing loss will occur when dose reaches $60 \mathrm{mg} / \mathrm{m}^{2}$ with high frequency. ${ }^{[26]}$ Moreover, the pathological manifestations of cisplatin ototoxicity are similar to those of aminoglycoside antibiotics, mainly damaging the cochlea and involved vestibule. ${ }^{[27]}$

Unfortunately, there is no specific measure to treat cisplatin ototoxicity mainly due to the fact that cisplatin-induced ototoxicity is irreversible. However, according to the mechanism of cisplatin ototoxicity, it can be reasonably prevented. Cisplatin ototoxicity could be guarded against by avoiding the generation of reactive oxygen species (ROS), neutralizing toxic products and inhibiting apoptosis. ${ }^{[28]}$ Recent studies have shown that ursolic acid, ${ }^{[29]} \mathrm{KL} 1333,{ }^{[30]}$ zingerone, ${ }^{[31]} \mathrm{N}$-acetylcysteine, ${ }^{[32]}$ intratympanic diltiazem-chitosan hydrogel, ${ }^{[33]}$ curcumin and ferulic acid $^{[34]}$ had protective effects on cisplatin-induced ototoxicity, but only in model animals or in vitro.

\section{Neurotoxicity}

Considering that cisplatin-induced neurotoxicity is dose-dependent, generally, when the cumulative dose of cisplatin exceeds $300-350 \mathrm{mg} / \mathrm{m}^{2},{ }^{[35]}$ patients begin to have symptoms, including sensory abnormalities, numbness, disappearance of tendon reflex, muscle spasm, decreased vibration sense, fine touch and proprioception sensitivity, also ataxia and Lhermitte's disease. At the cumulative dose of $500-600 \mathrm{mg} / \mathrm{m}^{2}$, almost all patients suffer neuropathy. ${ }^{[6]}$ It is 
difficult for cisplatin to cross the blood-brain barrier, but it has a high affinity with peripheral nerves, ${ }^{[37]}$ explaining why cisplatin-induced neurotoxicity mainly manifests as peripheral sensory neuropathy. ${ }^{[38]}$

The neurologic diseases caused by cisplatin are related to single dose and total dose. The scheme with same total dose but smaller single dose can prevent or alleviate sensory nerve damage. However, small doses of cisplatin therapy still could give rise to neurotoxicity. Therefore, it is necessary to find suitable drugs to resist the cisplatin-induced neurotoxicity. In recent years, researchers have discovered a considerable number of drugs against cisplatin-induced neurotoxicity, such as nucleolin, ${ }^{[39]}$ thymoquinone and geraniol, ${ }^{[40]}$ caffeic acid phenethyl ester (CAPE),${ }^{[41]}$ allantoin, ${ }^{[42]}$ sitagliptin, ${ }^{[43]}$ curcumin nanoparticles ${ }^{[44]}$ and sulfur-containing amino acids. ${ }^{[45]}$ Although these drugs mentioned above have shown good therapeutic prospects in antagonizing the neurotoxicity of cisplatin, they have not yet become conventional therapy against cisplatininduced neurotoxicity.

\section{Gastrointestinal reactions}

Gastrointestinal reaction includes nausea, vomiting, anorexia and diarrhea, frequently occurring within $1-6 \mathrm{~h}$ after administration, and the longest is no more than 24-48 $\mathrm{h}$. Although symptoms can be recovered after drug withdrawal, many patients refused to take cisplatin because of serious gastrointestinal reactions caused by chemotherapy. However, ondansetron has dramatically reduced the nausea and vomiting caused by cisplatin, moreover, it does not (or rarely) cause other adverse reactions due to its use. Clinically, 5-HT3 antagonists are mainly used to counteract gastrointestinal reaction. ${ }^{[46]}$ The latest research showed that XBXT, ${ }^{[47]}$ astaxanthin $^{[48]}$ and dokhwaljihwang-tang ${ }^{[49]}$ could effectively resist gastrointestinal reaction only in rats. It is worth noting that moxa salt packets combined with tropisetron hydrochloride can effectively reduce cisplatin chemotherapy-induced gastrointestinal reaction in clinic. ${ }^{[50]}$

\section{Resistance to Cisplatin}

Long term application of cisplatin will lead to drug resistance, one of the main reasons for stopping cisplatin during chemotherapy. Once the cells are resistant to cisplatin, the dose of the drug should be increased, simultaneously bringing about severe multiple organ toxicity. ${ }^{[1]}$ Once drug resistance occurs, other effective treatment options as well as the prognosis of patients will be limited. Therefore, how to reverse drug resistance has become a research big hit. Cisplatin resistance is caused by multiple resistance mechanisms, including accumulation of cisplatin, cisplatin inactivation induced by glutathione and metallothionein, and DNA repair. When multiple mechanisms work at the same time, cell resistance will occur, which is called multifactor resistance.

\section{Accumulation of cisplatin}

In cisplatin resistant cell lines, a decrease in drug accumulation is usually observed. The mechanism of accumulation reduction may include decreased uptake or increased export, or both.

(i) Decreased uptake. Previous studies have found that cisplatin mainly enters cells through membrane transporters, explaining why low levels of cisplatin are usually associated with low levels of transporters. ${ }^{[52]}$ The uptake of cisplatin is accomplished by copper transporters CTR 1 and CTR2 along with organic cation transporter (OCT) family. ${ }^{[53]}$

(ii) Increased export. It is generally believed that cisplatin is exported from cells via membrane transporters. Two transporters are involved in the clearance of cisplatin from cells:
P-type ATPase transporters or ATP-binding cassette transporters. ${ }^{[52]}$ Once the quantity of the two transporters increase, cisplatin efflux increases correspondingly.

\section{Cisplatin inactivation induced by glutathione and metallothionein}

GSH (glutathione), the most abundant intracellular mercaptan, combines with cysteine to form a peptide bond that could react with aminocaproic acid, in the metabolic synthesis of GSH. The concentration of GSH can be reduced by the use of butylthionine sulfoxide amine (BSO) because it is an inhibitor of $\mathrm{Y}$-glutamylcysteine synthetase. Elevated GSH levels have been found in many cisplatin resistant cell lines. Cisplatin can covalently bind to GSH after a nucleophilic attack on cells, and the resulting complex can be exported out of the cell through the ATP-dependent pump. The covalent binding with $\mathrm{GSH}$ inhibits the transformation of its monomer adducts to cross-linked adducts, thus greatly reducing the cytotoxicity of cisplatin. GSH could combine with cisplatin at a ratio of $2: 1$ to form a low toxic GS-Pt complex with increasing solubility, bringing about the inactivation of cisplatin. ${ }^{[52]}$ Moreover, GSH may be employed as a factor for the prediction of cellular resistance to cisplatin mainly due to the fact that $\mathrm{IC}_{50}$ of cisplatin in different cells seems to have a relation with mean of $\mathrm{GSH}$ level in 90 min. ${ }^{[54]}$ It is worth noting that three predominant biological thiols (cysteine, homocysteine and glutathione) can reduce cisplatin-based platinum (IV) prodrug. ${ }^{[55]}$

On the other hand, the combination of metallothionein proteins and cisplatin can also give rise to drug inactivation. MT (metallothionein), a series of small molecular proteins rich in cysteine, has high affinity with heavy metals such as zinc, copper, cadmium, and so on, explaining why MT can detoxify heavy metals. ${ }^{[52]}$ Thus, these proteins can bind to cisplatin, causing drug inactivation.

\section{DNA repair}

(i) MMR (Mismatch repair). Mismatch repair (MMR) refers to repair way to restore the normal nucleotide sequence in DNA molecules containing mismatch bases; mismatch repair process mainly employed to correct the mismatch on the DNA double helix needs to distinguish between the mother chain and sub-chain, so that only wrong nucleotides on sub-chain are removed, but not normal nucleotides on the mother chain. DNA damage induced by cisplatin can be detected by MMR system. MMR system-related proteins that recognize GpG adducts are $\mathrm{MSH} 2$ and MLH1. MMR system does not have the function of repairing DNA damage, but it can transmit pro-apoptotic signals. Kamal and co-workers ${ }^{[56]}$ found that the prognosis of surgical resection of non-small cell lung cancer patients with high expression of MSH2 without drug treatment was better than that of patients with low expression of MSHD. Therefore, in some clinical situations, high DNA repair ability can protect patients from tumor recurrence but damage at the same time.

(ii) TLS (Translesion synthesis). Trans-injury DNA synthesis, also known as damage bypass, is a kind of DNA synthesis that employs damaged nucleotides as a template and mixes bases into the replication termination site through DNA polymerase, thus delaying the repair of DNA. It is another mechanism newly discovered in recent years to participate in DNA repair. TLS includes error-free lesion bypass and error-prone lesion bypass that can lead to DNA damage-induced mutation. The mechanism by which cells can tolerate DNA damage is translesion synthesis (TLS). ${ }^{[57]}$ TLS is performed by a special group of DNA polymerase, TLS polymerase can bypass DNA damage and participate in the tolerance process, in mammals: POLH, POLI, POLK, REVI, REV3 and REV7, where POLH and REV3-REV7 heterodimers can bypass cisplatin-CG adducts to 
continue the synthesis of DNA. ${ }^{[58]}$

(iii) NER (Ncleotide Ecision Rpair). NER generally plays an important role in the repair of DNA bulk covalent damage, including DNA damage caused by UV dimer, polycyclic aromatic hydrocarbons, cisplatin and other heavy metals. The removal of cisplatin adducts from DNA is the most important process of nucleotide excision repair. When the plasmid DNA is destroyed by cisplatin, cells are transformed into wild-type, uvrA or uvrB cells. This may indicate that in this transition, genome damage caused by cisplatin can be repaired by nucleotide excision to reduce the damage. Previous studies have shown that DNA repair is related to cisplatin resistance. When cisplatin reacts with DNA, cells must clear or tolerate the damage to stand up to therapeutic effect; otherwise, DNA damage induced by cisplatin will cause a large number of cell death. ${ }^{[59]}$ Once inside the cell, cisplatin binds to DNA with the formation of Pt-DNA adducts, resulting in substantial kinking of the DNA. ${ }^{[52]}$ It is generally believed that these kinks can be identified and repaired by NER pathway, which explains why cells that lack DNA repair capabilities are highly sensitive to cisplatin. ${ }^{60]}$

Although various attempts have been made to reverse cisplatin resistance, so far, the success cases were extremely limited due to the multiple factors of cisplatin resistance. The sensitivity to cisplatin can be promoted by employing drugs that could increase cisplatin accumulation, reduce GSH or inhibit DNA repair. For instance, the combination of cisplatin with DNA repair inhibitors such as E-X PPI2, ${ }^{[61]} \mathrm{FdCyd}^{[62]}$ AZD6738, ${ }^{[63]}$ NU7026, ${ }^{[64]}$ B02 $2^{[65]}$ and compound $4 / 5^{[66]}$ can effectively increase the sensitivity of cisplatin.

\section{Conclusion}

As one of the most successful anticancer drugs, cisplatin has been utilized all over the world for more than 40 years. Although cisplatin has a good therapeutic effect on a variety of cancers, unfortunately, its side effects and drug resistance greatly limit its application. Moreover, the mechanism of cisplatin in human body has not been fully elucidated. Once the mechanism of cisplatin is clarified, it will be of great help to improve the efficacy of chemotherapy for cancer patients and provide a more effective scheme for the prevention and treatment of cancer.

\section{Acknowledgment}

We thank the Fund of Science and Technology Agency of Chengdu (No. 2019-YF09-00049-SN), the Open Project Program of Irradiation Preservation Technology Key Laboratory of Sichuan Province, Sichuan Institute of Atomic Energy (No. FZBC2020002), the Fundamental Research Funds for the Central Universities, the Sichuan Science and Technology Program (Nos. 2018HH0090, 2019YFH0032 and 2019YJ0548), the Key project of Sichuan Traditional Chinese Medicine Administration (No. 2018HJZX024), and the 14th Personalized Experimental Project (Nos. GX2020160034, GX2020160035).

\section{Conflict of Interest}

The authors declare no conflict of interest.

Copyright (C) 2021 Zhonglie Yang, Li Lin, Xiaoyan Peng, Xinyi Yu, Xiaobin Zhang, Hezhong Jiang, and Jiahong Li. This article is an open access article distributed under the terms and conditions of the Creative Commons Attribution (CC BY) license (http://creativecommons.org/ licenses/by/4.0/). The use, distribution or reproduction in other forums is permitted, provided the original author(s) or licensor are credited and that the original publication in this journal is cited, in accordance with accepted academic practice. No use, distribution or reproduction is permitted which does not comply with these terms.

\section{References}

[1] Peyrone, M. Ueber die Einwirkung des Ammoniaks auf Platinchlorür. Ann. Chem. Pharm. 1844, 51, 1.

[2] Werner, A. Beitrag zur Konstitution anorganischer Verbindungen. Z. Anorg. Chem. 1893, 3, 267.

[3] Rosenberg, B.; Vancamp, L. Platinum Compounds: a New Class of Potent Antitumour Agents. Nature 1969, 222, 385-386.

[4] Lebwohl, D.; Canetta, R. Clinical development of platinum complexes in cancer therapy: An historical perspective and an update. Eur. J. Cancer 1998, 34, 1522-1534.

[5] Cleare, M. J.; Hoeschele, J. D. Anti-tumour Platinum Compounds. Relationship between Structure and Activity. Plat. Met. Rev. 1973, $17,3$.

[6] Cleare, M. J.; Hoeschele, J. D. Studies on the antitumor activity of group VIII transition metal complexes. Part I. Platinum (II) complexes. Bioinorg. Chem. 1973, 2, 187.

[7] Jamieson, E. R.; Lippard, S. J. Structure, Recognition, and Processing of CisplatinDNA Adducts. Chem. Rev. 1999, 99, 2467-2498.

[8] Previati, M.; Lanzoni, I.; Corbacella, E. RNA expression induced by cisplatin in an organ of Corti-derived immortalized cell line. Hear. Res. 2004, 196, 8-18.

[9] Simpson, P. V.; Desai, N. M.; Casari, I. Metal-based antitumor compounds: Beyond cisplatin. Future Med. Chem. 2019, 11, 119-135.

[10] Lazarevic, T.; Rilak, A.; Bugarcic, Z. D. Platinum, palladium, gold and ruthenium complexes as anticancer agents: Current clinical uses, cytotoxicity studies and future perspectives. Eur. J. Med. Chem. 2017, 142, 8-31.

[11] Kasparkova, J.; Vojtiskova, M.; Natile, G. Unique Properties of DNA Interstrand Cross-Links of Antitumor Oxaliplatin and the Effect of Chirality of the Carrier Ligand. Chem.-Eur. J. 2008, 14, 13301341.

[12] Sigel, A.; Riddell, I. A.; Lippard, S. J. Cisplatin and Oxaliplatin: Our Current Understanding of Their Actions. Met. Ions Life 2018, 1-42.

[13] Arany, I.; Safirstein, R. L. Cisplatin Nephrotoxicity. Semin. Nephrol. 2003, 23, 460-464.

[14] Smith, B. D.; Smith, G. L.; Hurria, A. Future of cancer incidence in the United States: burdens upon an aging, changing nation. J. Clin. Oncol. 2009, 27, 2758-2765.

[15] Duan, Z.; Cai, G.; Li, J. Cisplatin-induced renal toxicity in elderly people. Ther. Adv. Med. Oncol. 2020, 12, 1758835920923430.

[16] Higuchi, K.; Yanagawa, T. Evaluating dose of cisplatin responsible for causing nephrotoxicity. PLoS One 2019, 14, e0215757.

[17] Dana, R.; Kachhwaha, V. S. Comparison of oral and intravenous hydration and diuretic, choice for protecting cisplatin induced nephrotoxicity. Indian. J. Cancer 1997, 33, 168-170.

[18] Li, Q.; Guo, D.; Dong, Z. Ondansetron Can Enhance CisplatinInduced Nephrotoxicity via Inhibition of Multiple Toxin and Extrusion Proteins (MATEs). Toxicol. Appl. Pharm. 2013, 273, 100-109.

[19] Un, H. R.; Ugan, R. A.; Kose, D. A novel effect of Aprepitant: Protection for cisplatin-induced nephrotoxicity and hepatotoxicity. Eur. J. Pharmacol. 2020, 880, 9.

[20] Abdel-Daim, M. M.; Mahmoud, O. M.; Al Badawi, M. H. Protective effects ofCitrus limoniaoil against cisplatin-induced nephrotoxicity. Environ. Sci. Pollut. Res. 2020, 11.

[21] Li, C.; Li, L. P.; Yi, Y. D. L-tetrahydropalmatine attenuates cisplatininduced nephrotoxicity via selective inhibition of organic cation transporter 2 without impairing its antitumor efficacy. Biochem. Pharmacol. 2020, 177, 11.

[22] Khalifa, E. A.; Ahmed, A. N.; Hashem, K. S. Therapeutic Effects of the Combination of Alpha-Lipoic Acid (ALA) and Coenzyme Q10 (CoQ10) on Cisplatin-Induced Nephrotoxicity. Int. J. Inflamm. 2020, 2020, 11.

[23] Kolinsky, D. C.; Hayashi, S. S.; Karzon, R. Late Onset Hearing 
Loss: A Significant Complication of Cancer Survivors Treated With Cisplatin Containing Chemotherapy Regimens. J. Pediat. Hematol. Onc. 2010, 32, 119.

[24] Gentilin, E.; Simoni, E.; Candito, M. Cisplatin-Induced Ototoxicity: Updates on Molecular Targets. Trends Mol. Med. 2019, 25, $1123-1132$.

[25] Turan, C.; Kantar, M.; Aktan, C. Cisplatin ototoxicity in children: risk factors and its relationship with polymorphisms of DNA repair genes ERCC1, ERCC2, and XRCC1. Cancer Chemother. Pharmacol. 2019, 84, 1333-1338.

[26] Rademaker-Lakhai, J. M. Relationship between cisplatin administration and the development of ototoxicity. J. Clin. Oncol. 2006, 24, 918

[27] Oh, S. H.; Yu, W. S.; Song, B. H. Expression of Heat Shock Protein 72 in Rat Cochlea with Cisplatin-induced Acute Ototoxicity. Acta Oto-Laryngol. 2000, 120, 146-150.

[28] Rybak, L. P.; Whitworth C.; Somani, S. Application of Antioxidants and Other Agents to Prevent Cisplatin Ototoxicity. Laryngoscope 2010, 109, 1740-1744.

[29] Di, Y.; Xu, T.; Tian, Y. Ursolic acid protects against cisplatininduced ototoxicity by inhibiting oxidative stress and TRPV1mediated $\mathrm{Ca}^{2+}$-signaling. Int. J. Mol. Med. 2020, 46, 806-816.

[30] Lee, H. S.; Kim, Y. R.; Lee, I. K. KL1333, a derivative of betalapachone, protects against cisplatin-induced ototoxicity in mouse cochlear cultures. Biomed. Pharmacother. 2020, 126, 6.

[31] Lee, C. H.; Lee, D. H.; Lee, S. M. Otoprotective Effects of Zingerone on Cisplatin-Induced Ototoxicity. Int. J. Mol. Sci. 2020, $21,11$.

[32] Somdas, M. A.; Gunturk, I.; Balcioglu, E. Protective effect of $\mathrm{N}$-acetylcysteine against cisplatin ototoxicity in rats: a study with hearing tests and scanning electron microscopy. Braz. J. Otorhinolaryngol. 2020, 86, 30-37.

[33] Naples, J. G.; Ruckenstein, M. J.; Singh, J. Intratympanic Diltiazem-Chitosan Hydrogel as an Otoprotectant Against Cisplatin-Induced Ototoxicity in a Mouse Model. Otol. Neurotol. 2020, 41, 115-122.

[34] Paciello, F.; Rita Fetoni, A.; Mezzogori, D. The dual role of curcumin and ferulic acid in counteracting chemoresistance and cisplatin-induced ototoxicity. Sci. Rep. 2020, 10, 1063.

[35] Santos, N.; Ferreira, R. S.; Santos, A. C. D. Overview of cisplatininduced neurotoxicity and ototoxicity, and the protective agents. Food Chem. Toxicol. 2020, 136, 111079

[36] Troy, L.; McFarland, K.; Littman-Power, S. Cisplatin-based therapy: A neurological and neuropsychological review. Psycho-Oncol. 2000, 9, 29.

[37] Fischer, D.; Malik, T. Chemotherapy-Induced Peripheral Neuropathy. In Practical Chronic Pain Management: A Case-Based Approach. Ed.: Malik, T., Springer International Publishing, 2020, pp. 371-380.

[38] Qi, L.; Luo, Q.; Zhang, Y. Advances in Toxicological Research of the Anticancer Drug Cisplatin. Chem. Res. Toxicol. 2019, 32, 1469-1486.

[39] Podratz, J. L.; Tang, J. J.; Polzin, M. J. Mechano growth factor interacts with nucleolin to protect against cisplatin-induced neurotoxicity. Exp. Neurol. 2020, 331, 113376.

[40] Kandeil, M. A.; Mahmoud, M. O.; Abdel-Razik, A. R. H. Thymoquinone and geraniol alleviate cisplatin-induced neurotoxicity in rats through downregulating the p38 MAPK/STAT-1 pathway and oxidative stress. Life Sci. 2019, 228, 145-151.

[41] Ferreira, R. S.; dos Santos, N. A. G.; Bernardes, C. P. Caffeic Acid Phenethyl Ester (CAPE) Protects PC12 Cells Against Cisplatin-Induced Neurotoxicity by Activating the AMPK/SIRT1, MAPK/Erk, and PI3K/Akt Signaling Pathways. Neurotox. Res. 2019, $36,175-192$.

[42] N S, V.; Mohamad, A.; Razdan, R. Allantoin attenuates deficits of behavioural and motor nerve conduction in an animal model of cisplatin-induced neurotoxicity in rats. Anim. Models Exp. Med.
2019, 2, 114-120.

[43] Li, Y. X.; Zheng, M. Y.; Sah, S. K. Neuroprotective influence of sitagliptin against cisplatin-induced neurotoxicity, biochemical and behavioral alterations in Wistar rats. Mol. Cell. Biochem. 2019, 455, 91-97.

[44] Khadrawy, Y. A.; Ei-Gizawy, M. M.; Sorour, S. M. Effect of curcumin nanoparticles on the cisplatin-induced neurotoxicity in rat. Drug Chem. Toxicol. 2019, 42, 194-202.

[45] Rosic, G.; Joksimovic, J.; Selakovic, D. The Beneficial Effects of Sulfur-containing Amino Acids on Cisplatin-induced Cardiotoxicity and Neurotoxicity in Rodents. Curr. Med. Chem. 2018, 25, 391-403.

[46] Cubeddu, L. X.; Hoffmann, I. S.; Fuenmayor, N. T.; Finn, A. L. Efficacy of Ondansetron ( $\mathrm{Gr} 38032 \mathrm{~F})$ and the Role of Serotonin in Cisplatin-Induced Nausea and Vomiting. New Eng. J. Med. 1990, 322, 810-816.

[47] Li, G.; Zhang, Q.; Du, J. Effect of XBXT on Prevention and Treatment of Gastrointestinal Tract Inflammation Injury Induced by Cisplatin in Rats. Traditional Chinese Drug Research \& Clinical Plarmacology 2017, 28, 459-463.

[48] Yilmaz, Y.; Tumkaya, L.; Mercantepe, T. Protective effect of astaxanthin against cisplatin-induced gastrointestinal toxicity in rats. Eur. Surg. 2020, 7.

[49] Hyun, L. The Effects of Dokhwaljihwang-tang Intravenous Pharmacopuncture on Cisplatin-Induced Emesis and Gastrointestinal Mobility Disorder in Rats. J. Acupunct. Res. 2017, 34, 39-48.

[50] Cai, Y.; Wu, Y.; Ye, F. Moxa salt packets at Zhongwan (CV 12) for cisplatin chemotherapy-induced gastrointestinal reactions: a clinical study. Chinese Acupuncture \& Moxibustion 2016, 36, 405-408.

[51] Brabec, V.; Kasparkova, J. Modifications of DNA by platinum complexes. Relation to resistance of tumors to platinum antitumor drugs. Drug Resist. Update 2005, 8, 131-146.

[52] Amable, L. Cisplatin resistance and opportunities for precision medicine. Pharmacol. Res. 2016, 106, 27-36.

[53] Hall, M. D.; Okabe, M.; Shen, D.-W. The Role of Cellular Accumulation in Determining Sensitivity to Platinum-Based Chemotherapy. Annu. Rev. Pharmacol. Toxicol. 2008, 48, 495-535.

[54] Jamalia, B.; Nakhjavani, M.; Hosseinzadeh, L. Intracellular GSH Alterations and Its Relationship to Level of Resistance following Exposure to Cisplatin in Cancer Cells. Iran. J. Pharm. Res. 2015, $14,513-519$

[55] Wan, W. F.; Sun, J. J.; Liu, W. R. Reductions of the cisplatin-based platinum(IV) prodrug cis, cis, trans- $\mathrm{Pt}\left(\mathrm{NH}_{3}\right)_{2} \mathrm{Cl}_{2} \mathrm{Br}_{2}$ by predominant biological thiols: kinetic and mechanistic studies. Transit. Met. Chem. 2019, 44, 535-544.

[56] Kama, N. S.; Soria, J. C.; Mendiboure, J. MutS Homologue 2 and the Long-term Benefit of Adjuvant Chemotherapy in Lung Cancer. Clin. Cancer Res. 2010, 16, 1206.

[57] Bassett, E.; Vaisman, A.; Tropea, K. A.Frameshifts and deletions during in vitro translesion synthesis past Pt-DNA adducts by DNA polymerases $\beta$ and $\eta$ DNA Repair 2002, 1, 1003.

[58] Shachar, S.; Ziv, O.; Avkin, S. Two-polymerase mechanisms dictate error-free and error-prone translesion DNA synthesis in mammals. EMBO J. 2009, 28, 383.

[59] Rocha, C. R. R.; Silva, M. M.; Quinet, A. DNA repair pathways and cisplatin resistance: an intimate relationship. Clinics 2018, 73, e478s.

[60] Martin, L. P.; Hamilton, T. C.; Schilder, R. J. Platinum resistance: the role of DNA repair pathways. Clin. Cancer Res. 2008, 14, 1291-1295.

[61] McNeil, E. M.; Astell, K. R.; Ritchie, A.-M. Inhibition of the ERCC1-XPF structure-specific endonuclease to overcome cancer chemoresistance. DNA Repair 2015, 31, 19-28.

[62] Kelley, M. R.; Logsdon, D.; Fishel, M. L. Targeting DNA repair 
pathways for cancer treatment: what's new? Future Oncol. 2014, 10, 1215-1237.

[63] Vendetti, F. P.; Lau, A.; Schamus, S. The orally active and bioavailable ATR kinase inhibitor AZD6738 potentiates the anti-tumor effects of cisplatin to resolve ATM-deficient non-small cell lung cancer in vivo. Oncotarget 2015, 6, 44289-44305.

[64] Albarakati, N.; Abdel-Fatah, T. M. A.; Doherty, R. Targeting BRCA1-BER deficient breast cancer by ATM or DNA-PKcs blockade either alone or in combination with cisplatin for personalized therapy. Mol. Oncol. 2015, 9, 204-217.

[65] Alagpulinsa, D. A.; Ayyadevara, S.; Shmookler Reis, R. J. A Small-Molecule Inhibitor of RAD51 Reduces Homologous
Recombination and Sensitizes Multiple Myeloma Cells to Doxorubicin. Front. Oncol. 2014, 4

[66] Sail, V.; Rizzo, A. A.; Chatterjee, N. Identification of Small Molecule Translesion Synthesis Inhibitors That Target the Rev1-CT/RIR Protein-Protein Interaction. ACS Chem. Biol. 2017, 12, 1903-1912.

Received December 4, 2020 Accepted January 30, 2021 